\title{
A gravidade da cárie está associada à desnutrição proteico-calórica em pré-escolares?
}

\author{
Is the severity of caries associated with malnutrition \\ in preschool children?
}

Cecilia Claudia Costa Ribeiro ${ }^{1}$

Mariana Carvalho Batista da Silva ${ }^{2}$

Camila Maiana Pereira Machado ${ }^{3}$

Marizélia Rodrigues Costa Ribeiro ${ }^{4}$

Érika Bárbara Abreu Fonseca Thomaz ${ }^{1}$

\footnotetext{
${ }^{1}$ Programa de PósGraduação em Saúde Coletiva, Universidade Federal do Maranhão (UFMA). Av. dos Portugueses s/n, Campus do Bacanga. 65.085-582 São Luís MA Brasil. cecilia _ribeiro@hotmail.com ${ }^{2}$ Curso de Odontologia, UNICEUMA ${ }^{3}$ Curso de Odontologia, UFMA.

${ }^{4}$ Departamento de Medicina III, UFMA.
}

Abstract An analysis was conducted to evaluate if the severity of caries is associated with proteincalorie malnutrition in preschool children. The cross-sectional study was performed on a retrospective cohort of 625 children aged 24-71 months attending daycare centers in São Luis, Maranhão. A hierarchical model was evaluated at five levels: 1.) Socio-economic variables (economic group, mother's educational level and skin color); 2) Access variables; 3) Low birth weight (LBW) and exclusive breastfeeding; 4) Weight per height at 12 months; and 5) Severity of caries and albumin (Alb) levels. The outcome was protein-calorie malnourishment (weight per height $z$ score $<$ -2). The prevalence of outcome was $5.0 \%$ and the prevalence of caries was 32\%. At the first level, the variables were not associated with the outcome, but the economic group was maintained until the final model. The variables in the second and third levels were not significant. At the fourth level, weight for height at 12 months was not significant, but was maintained until the final model. In the last level, severity of caries was positively associated with malnourishment. The association between severity of caries and malnourishment suggest that oral health should be integrated with public health care policies for children.

Key words Protein-calorie malnutrition, Dental caries, Preschoolers
Resumo Avaliou-se se gravidade da cárie está associada à desnutrição proteico-calórica em préescolares. Foi realizado um estudo seccional aninhado a uma coorte retrospectiva de 625 crianças entre 24 -71 meses, em São Luís, Maranhão. Um modelo hierarquizado foi avaliado em cinco níveis: $1^{\circ}$ ) Variáveis socioeconômicas (classe econômica, escolaridade materna, cor da pele); $2^{\circ}$ ) Variáveis de acesso; 30) Baixo peso ao nascer e amamentação exclusiva; 4º) Peso para altura aos 12 meses e 5o) Gravidade da cárie e níveis de albumina (Alb). O desfecho foi desnutrição proteico-calórica (z escore altura para idade < -2). A prevalência do desfecho foi de 5,0\%, e da cárie foi de $32 \%$. As variáveis do primeiro nível não foram associadas ao desfecho, porém a classe econômica foi mantida até o modelo final. As variáveis do segundo e do terceiro níveis não foram significativas. No quarto nível, peso para altura aos 12 meses não foi significativo, porém foi mantido até o modelo final. No último nível, os níveis de Alb não foram associados ao desfecho e a gravidade da doença cárie foi positivamente associada com desnutrição proteico-calórica em pré-escolares. A associação da gravidade da cárie com déficit antropométrico sinaliza que a saúde bucal deva ser parte importante nas políticas públicas de atenção à infância.

Palavras-chave Desnutrição proteico-calórica, Cárie dentária, Pré-escolar 
Introdução

A prevalência de desnutrição proteico-calórica, definida como "[...] deficiência energética e proteica com redução da massa muscular e adiposa [...]"', foi reduzida em crianças brasileiras menores de cinco anos, ao longo das últimas décadas $^{2}$. Na última Pesquisa Nacional de Demografia e Saúde da Criança e da Mulher (PNDS 2006), apenas $1,9 \%$ das crianças nessa faixa etária apresentavam déficits de peso para altura ${ }^{2}$.

Entretanto, a desnutrição proteico-calórica ainda representa um problema de saúde públi$\mathrm{ca}^{3,4}$, posto que os danos sofridos no início da vida podem levar ao comprometimento permanente do indivíduo, inclusive afetando futuras gerações ${ }^{3}$. Além disso, verifica-se que os casos de desnutrição infantil crônica no Brasil acumulamse em municípios da região Nordeste ${ }^{5,6}$, evidenciando a persistência de iniquidades sociais em saúde.

Fatores pré-natais ${ }^{4}$, intrauterinos ${ }^{3}$ e pós-natais ${ }^{7}$ têm sido associados à desnutrição proteicocalórica. Determinantes sociais, políticos e culturais são apontados como causas distais da desnutrição infantil porque influenciam os cuidados à saúde da criança e sua forma de adoecer ${ }^{7,8}$. Nessa perspectiva, a cárie na infância também possui um claro componente social ${ }^{9-13} \mathrm{com}$ maior prevalência em situações de desigualdade social, tanto em países desenvolvidos quanto nos em desenvolvimento ${ }^{12-15}$.

Quanto à associação entre cárie em crianças e desnutrição proteico-calórica, o acesso a alimentos de menor custo, os quais frequentemente são pobres em nutrientes e ricos em açúcares, pode representar um fator comum mais proximal àquelas alterações, já que práticas alimentares inadequadas estão presentes tanto em quadros de desnutrição ${ }^{7}$, quanto na cárie em crianças ${ }^{16,17}$.

Ademais, o não tratamento das cavidades profundas de cárie dentária, as quais geralmente são responsáveis por quadros de dor ${ }^{18} \mathrm{e}$ dificuldades de mastigação ${ }^{19}$, também podem resultar em má nutrição ${ }^{20}$ e crescimento inadequado da criança $\mathrm{a}^{19,21}$.

A associação entre alterações nutricionais e cárie em crianças tem sido alvo de recentes estudos, mostrando resultados conflitantes. Enquanto alguns estudos apontaram uma associação entre cárie na infância e déficits antropométricos, quer em estatura (comprimento ou altura) ${ }^{22}$, quer no índice de massa corporal ${ }^{23}$, quer ainda no peso ${ }^{24-28}$, outros não conseguiram estabelecer qualquer associação entre estado nutricional e cárie eq-32 $^{20}$.
Nesse contexto, este estudo se propôs a avaliar se a gravidade da doença cárie está associada à desnutrição proteico-calórica em pré-escolares em uma abordagem hierarquizada, ajustando o modelo para clássicos confundidores dessa deficiência nutricional.

\section{Metodologia}

\section{Desenho e área do estudo}

Trata-se de um estudo transversal aninhado a uma coorte retrospectiva em crianças de 24 a 71 meses residentes no município de São Luís, Maranhão. Esta população não tem abastecimento de água fluoretada pública regular ${ }^{33}$ e a assistência odontológica pública para as crianças é escassa ${ }^{34}$.

\section{Caracterização e delineamento da amostra}

As crianças foram recrutadas em 14 creches comunitárias, nos diferentes distritos sanitários do município de São Luís. Todas as crianças foram convidadas a participar do estudo. Foram elegíveis crianças matriculadas nas creches com no máximo 71 meses de idade e que tivessem todos os dentes decíduos erupcionados.

O tamanho mínimo da amostra com perdas foi estimado em 530 crianças, considerando a prevalência de cárie de $50 \%$ para as crianças expostas aos fatores de risco e $35 \%$ para as não expostas, adotando-se proporção de 1:1 entre expostos e não expostos, nível de significância de $5 \%$ e poder do teste igual a $80 \%$.

\section{Coleta de dados}

Dados sociodemográficos e econômicos e as informações sobre amamentação foram obtidos por meio de um questionário aplicado aos pais e/ou responsáveis. As medidas antropométricas do nascimento e aos 12 meses (peso e comprimento) foram consultadas na Caderneta de Saúde da Criança (CSC).

As crianças tiveram aferidas as medidas de peso corporal em quilogramas $(\mathrm{Kg})$, altura em centímetros $(\mathrm{cm})$ segundo técnicas padronizadas pela $\mathrm{OMS}^{35}$. Para a aferição da altura foi usado o Estadiômetro Portátil Alturexata ${ }^{\circledR}$ (Belo Horizonte, MG, Brasil) e para o peso, balança digital de plataforma Filizola ${ }^{\circledR}$ (São Paulo, SP, Brasil), com variação máxima de 100g. Para tal, dois examinadores foram treinados e calibrados 
no uso dos instrumentos por um médico pediatra. Todas as medidas foram feitas em separado e em duplicata, e a média das duas avaliações foi registrada como medida da criança. A concordância intraexaminador foi $0,89( \pm 0,01)$ e a interexaminadores, 0,91 pelo teste Kappa. A altura para idade (ai) foi expressa em escore z. Para cálculo deste índice foi utilizado o software WHO Anthro 3.2.2 ${ }^{36}$ para as crianças até 60 meses e WHO Anthro Plus $1.4 .0^{37}$ para crianças com mais de 60 meses.

O exame clínico de cárie foi realizado sob luz artificial por um único profissional calibrado (MCBS) de acordo com os critérios da Organização Mundial de Saúde (OMS) ${ }^{38}$ para cálculo do índice ceo-d (número de dentes cariados, indicados para extração e obturados). Também foi observada a presença de fístulas e raízes residuais que pudessem dificultar a mastigação das crianças. A concordância intraexaminador para índice ceo-d foi 0,92 pelo teste Kappa.

As coletas sanguíneas foram realizadas com as crianças em jejum entre as 8:00 e 9:00 da manhã. No exame hematológico foi avaliado o nível sérico de albumina (Alb). A concentração de Alb foi determinada pelo método colorimétrico.

\section{Modelo teórico}

O desfecho estudado foi desnutrição proteico-calórica, analisada pelo valor do Z escore Z altura para idade atual, tratado como uma variável dicotômica (escore $Z$ ai idade $<-2$ : sim, não). A altura para idade, segundo a Organização Mundial de Saúde (OMS) ${ }^{35}$, é um dos melhores preditores para avaliação da desnutrição crônica.

O referencial teórico para este estudo (Figura 1) foi construído em cinco níveis: primeiro, variáveis socioeconômicas; segundo, variáveis de acesso; terceiro, baixo peso ao nascer e amamentação exclusiva ; quarto, peso para altura aos 12 meses e, quinto, gravidade de cárie e níveis de albumina atuais.

O primeiro nível incluiu as variáveis socioeconômicas, como as mais distais no modelo teórico na associação com o desfecho, por serem capazes de influenciar todos os níveis seguintes. Para a classe econômica utilizou-se o Critério de Classificação Econômica Brasil da Associação Brasileira de Empresa de Pesquisa (ABEP) ${ }^{39}$. Para análise, as classes A1, A2, B1 e B2 foram agrupadas e renomeadas de classe Alta. As classes C1 e C2 agrupadas em classe Média e as classes D e E em Baixa. A cor da pele referida pelo responsável foi categorizada como branca e não branca (ne-

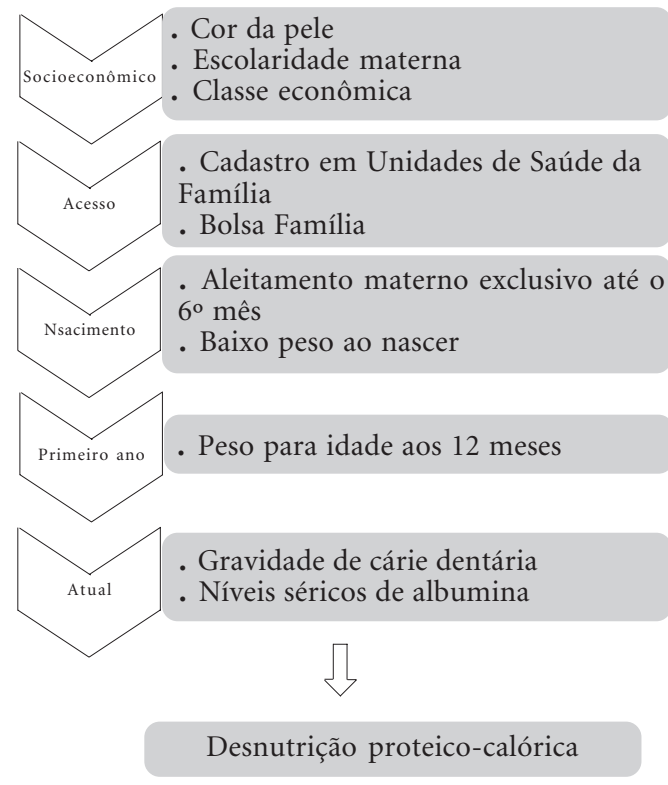

Figura 1. Quadro hierárquico das variáveis associadas com a desnutrição proteico-calórica.

gra ou parda). A escolaridade materna em anos de estudo ( $>8$ anos; $\leq 8$ anos).

No segundo nível foram incluídas as variáveis de acesso: cadastro em Unidade de Saúde da Família (USF) e inclusão no Programa Bolsa Família - PBF, ambas dicotomizadas (sim ou não).

No terceiro nível foram incluídas as variáveis: baixo peso ao nascer (BPN) e amamentação exclusiva até o $6^{\circ}$ mês de vida, dicotomizadas em sim e não. O BPN foi considerado menor que $2500 \mathrm{~g}^{40}$ de acordo com a informação da CSC.

No quarto nível foi incluído a variável peso aos 12 meses, classificado em magreza acentuada ( $<$ escore $z-3$ ), magreza ( $\geq$ escore $z-3$ e $<$ escore $\mathrm{z}-2$ ), eutrofia ( $\geq$ escore $\mathrm{z}-1$ e $<$ escore $\mathrm{z}$ +1 ), risco de sobrepeso ( $>$ escore $z+1$ e $\leq$ escore $z+2$ ), sobrepeso ( $>$ escore $z+2$ e $\leq$ escore $z+3)$ e obesidade ( $>$ escore $z+3$ ). Neste estudo, as categorias magreza e magreza acentuada foram agrupadas em magreza, e sobrepeso e obesidade, em obesidade.

O quinto nível correspondeu às variáveis gravidade de cárie e nível sérico de Alb, ambas tratadas como variáveis discretas. A gravidade da doença em cada criança correspondeu ao somatório dos dentes com lesões cariosas cavitadas, 
incluindo o número de dentes com fístula ou raiz residual, e tratada com variável discreta.

\section{Processamento e análise dos dados}

Utilizou-se análise de regressão logística, com modelagem forward, orientada por uma abordagem teórica hierarquizada.

Os modelos foram construídos seguindo um quadro teórico com cinco níveis já apresentados. As variáveis analisadas no primeiro nível foram inseridas e transferidas para os níveis subsequentes quando o valor de $\mathrm{p}<0,20$. No segundo nível, variáveis mantidas do primeiro nível e as variáveis que pertencem a este nível foram inseridos e transferidos para o nível seguinte se o valor de $\mathrm{p}$ fosse menor que 0,2 . O mesmo procedimento foi mantido até o último nível. Estimaram-se as razões de prevalência (RP) ajustadas e respectivos intervalos de confiança a 95\% (IC 95\%). O nível de significância foi fixado em 0,05 . Todas as análises estatísticas foram realizadas no programa STATA $10.0^{41}$.

\section{Aspectos éticos}

O Comitê de Ética em Pesquisa do Hospital Universitário da Universidade Federal do Maranhão (UFMA) aprovou o estudo. Os pais ou responsáveis legais das crianças assinaram um Termo de Consentimento Livre e Esclarecido (TCLE) permitindo a participação das crianças na pesquisa. Todas as crianças com alterações nos parâmetros nutricionais (déficits de albumina e medidas antropométricas) foram encaminhadas para médico pediatra para o tratamento adequado e aquelas com atividade da doença cárie ou necessidades de tratamento cirúrgico-restaurador foram encaminhadas para atendimento na Clínica de Odontopediatria da UFMA.

\section{Resultados}

A prevalência de desnutrição proteico-calórica foi de 5,0\% $(\mathrm{n}=34)$. A Tabela 1 mostra a distribuição das variáveis explanatórias do estudo de acordo com a altura para idade.

No nível 1 , as variáveis socioeconômicas não mostraram significância para o desnutrição proteico-calórica $(\mathrm{p}>0,05)$, porém a classe econômica foi transferida para o nível subsequente $(\mathrm{p}=$ 0,07). As variáveis de acesso inseridas no nível 2 não foram significantes. No nível 3 , nem o peso ao nascer ou amamentação exclusiva até 6 meses, foram associadas com desnutrição proteico-calórica $(\mathrm{p}>0,05)$. No nível 4 , o peso para altura aos 12 meses não foi significativo $(\mathrm{p}=0,07)$, porém apresentou $\mathrm{RP}=4,48$ (IC $0,88-22,83$ ). No último nível, a gravidade da doença cárie foi positivamente associada com desnutrição proteicocalórica ( $\mathrm{p}=0,042$; $\mathrm{RP}=1,17$; IC 1,05-1,36). Nenhuma criança do estudo apresentou deficiência de albumina ${ }^{42}$. Os níveis de Alb, tratados como variável contínua, não foram significativamente associados ao desfecho (Tabela 2).

\section{Discussão}

Registrou-se que a prevalência de desnutrição infantil crônica cresceu, em média, $17 \%$ com o aumento da gravidade da doença cárie, mesmo após ajuste para variáveis sociodemográficas, de acesso e de saúde ao nascimento e do primeiro ano de vida.

No nível 1, as variáveis socioeconômicas (classe econômica, escolaridade materna, cor da pele) não estiveram associadas à altura para a idade. Estes dados poderiam ser justificados por uma maior homogeneidade da população, predominando a escolaridade materna com ensino médio incompleto $(64,5 \%)$ e a classe econômica C $(67,4 \%)$; ainda, todas as crianças eram pertencentes a creches comunitárias, espaço normalmente utilizado por famílias de mais baixa ren$\mathrm{da}^{43}$. O fato de São Luís ser uma capital de grande miscigenação, a cor da pele não ser uma representação da raça ${ }^{44}$, nem mesmo exprimir a condição socioeconômica ${ }^{45}$, o que pode ter contribuído para a variável explanatória não ter sido associada ao desfecho no presente estudo.

Ter cadastro em USF não se mostrou como indicador de proteção à desnutrição proteicocalórica. Estes dados discordam dos achados de Lima et al. ${ }^{46}$, mostrando que crianças assistidas pelos programas de saúde, em especial a Estratégia de Saúde da Família (ESF), apresentaram um declínio do déficit de estatura, principalmente para população de menor renda. Dados da cobertura da ESF no Maranhão são precários e às vezes não representam a realidade da oferta de serviços de saúde, com repercussões no acesso à atenção básica. Há ainda indícios de que apesar da relativa cobertura da ESF em São Luís, permanece o modelo assistencial médico hegemônico medicalizador, curativista e pouco resoluti$\mathrm{vo}^{47}$. É possível que estes problemas locais possam reduzir a efetividade das ações na redução da desnutrição infantil. Foi identificado ainda que 
Tabela 1. Distribuição das variáveis de estudo de acordo com a altura para idade. São Luís. MA. Brasil, 2012.

\begin{tabular}{|c|c|c|c|}
\hline Variável & n (\%) & $\begin{array}{c}\text { Altura adequada } \\
\text { n }(\%)\end{array}$ & $\begin{array}{c}\text { Déficit de altura } \\
\mathbf{n}(\%)\end{array}$ \\
\hline \multicolumn{4}{|l|}{ Classe Econômica ABEPa } \\
\hline Alta & $70(11,2)$ & $65(92,8)$ & $5(7,2)$ \\
\hline Média & $421(67,4)$ & $398(94,5)$ & $23(5,5)$ \\
\hline Baixa & $134(21,4)$ & $131(97,8)$ & $3(2,2)$ \\
\hline \multicolumn{4}{|l|}{ Cor da Pele } \\
\hline Branca & $172(27,5)$ & $163(94,8)$ & $9(5,2)$ \\
\hline Não Branca & $453(72,5)$ & $431(95,2)$ & $22(4,8)$ \\
\hline \multicolumn{4}{|c|}{ Escolaridade materna (anos completos) } \\
\hline$<8$ anos & $403(64,5)$ & $384(64,6)$ & $19(35,4)$ \\
\hline$\geq 8$ anos & $222(35,5)$ & $210(61,3)$ & $12(38,7)$ \\
\hline \multicolumn{4}{|c|}{ Cadastro em Unidade de Saúde da Família } \\
\hline Sim & $267(42,7)$ & $251(42,2)$ & $16(51,6)$ \\
\hline Não & $358(57,3)$ & $343(57,8)$ & $15(48,4)$ \\
\hline \multicolumn{4}{|l|}{ Bolsa Família } \\
\hline Sim & $251(40,1)$ & $241(96,0)$ & $10(4,0)$ \\
\hline Não & $374(59,9)$ & $353(94,4)$ & $21(5,6)$ \\
\hline \multicolumn{4}{|l|}{ Baixo peso ao nascer $(n=489)$} \\
\hline $\operatorname{Sim}$ & $24(95,1)$ & $15(94,0)$ & $1(6,0)$ \\
\hline Não & $465(4,9)$ & $450(95,0)$ & $23(5,0)$ \\
\hline \multicolumn{4}{|l|}{ Amamentação exclusiva } \\
\hline Sim & $413(66,2)$ & $391(94,6)$ & $22(5,4)$ \\
\hline Não & $211(33,8)$ & $202(95,7)$ & $9(4,3)$ \\
\hline \multicolumn{4}{|c|}{ Peso aos 12 meses - ZPI $(\mathrm{n}=388)$} \\
\hline Adequado & $308(79,3)$ & $293(95,0)$ & $15(5,0)$ \\
\hline Abaixo do peso & $13(3,3)$ & $11(84,6)$ & $2(15,4)$ \\
\hline Acima do peso & $67(17,4)$ & $65(97,0)$ & $2(3,0)$ \\
\hline \multicolumn{4}{|l|}{ Cárie $^{\mathrm{b}}$} \\
\hline $\operatorname{Sim}$ & $189(30,2)$ & $173(91,5)$ & $16(8,5)$ \\
\hline Não & $436(69,8)$ & $421(96,0)$ & $15(4,0)$ \\
\hline \multicolumn{4}{|c|}{ Deficiência de Albumina $^{c}(\mathrm{n}=350)$} \\
\hline $\operatorname{Sim}$ & N.O & N.O & N.O \\
\hline Não & $350(100,0)$ & $333(95,0)$ & $17(5,0)$ \\
\hline
\end{tabular}

${ }^{a}$ Critério de Classificação Econômica ABEP ${ }^{39}$. ${ }^{b}$ Presença de cavitações. ${ }^{c}$ Albumina $<3,5$ (Blackburn et al. $\left.{ }^{42}\right)$. N.O = não observado.

receber o benefício bolsa família do governo também não se mostrou como uma variável de proteção à desnutrição proteico-calórica, ainda que $40 \%$ das crianças da amostra recebessem esse benefício do governo.

As variáveis relacionadas às medidas antropométricas ao nascer e ao primeiro ano de vida não estiveram associadas à altura para idade entre 24 e 71 meses. Estes representam fatores de risco mais clássicos à desnutriçãa ${ }^{3,4,48}$. É possível que o número de observações dessas variáveis explanatórias, bem como do desfecho, tenham contribuído para esses achados.

Um maior número de lesões de cárie foi positivamente associado ao déficit nutricional, con- cordando com achados de Ayhan et al. ${ }^{49}$ e Floyd et al. ${ }^{50}$, nos quais crianças acometidas por cárie apresentaram menor estatura, comparadas com crianças da mesma faixa etária livres da doença. Esta associação é plausível, haja vista que a cárie dentária pode resultar em dor provocada pela mastigação e pelo contato com alimentos ${ }^{51}$, o que poderia ter implicações na redução da ingestão alimentar e restrição energético-proteica e, consequentemente interferir no crescimento ${ }^{52}$.

Essa associação entre gravidade da cárie dentária e má-nutrição poderia ainda ocorrer por interação de riscos nos grupos de menor poder aquisitivo, haja vista que a situação pobreza poderia conduzir a problemas de saúde dental ${ }^{53}$, devido à 
Tabela 2. Análise de regressão de logística das variáveis associadas com a desnutrição proteica-calórica em crianças. São Luís. Maranhão. Brasil, 2012.

\begin{tabular}{|c|c|c|c|c|c|}
\hline \multirow[b]{2}{*}{ Variáveis } & Modelo 1 & Modelo 2 & Modelo 3 & Modelo 4 & Modelo 5 \\
\hline & RP (IC95\%) p & RP (IC95\%) p & $\mathrm{RP}(\mathrm{IC} 95 \%) \quad p$ & $\mathrm{RP}(\mathrm{IC} 95 \%) \quad p$ & RP (IC95\%) p \\
\hline \multicolumn{6}{|c|}{ Classe econômica $\mathrm{ABEP}^{\mathrm{a}}$} \\
\hline Baixa & 0,07 & 0,14 & 0,09 & 0,60 & 0,60 \\
\hline Média & $0,71(0,26-1,97)$ & $0,73(0,26-2,04)$ & $0,74(0,27-2,03)$ & $0,71(0,19-2,70)$ & $0,71(0,19-2,70)$ \\
\hline Alta & $0,24(0,14-1,12)$ & $0,30(0,69-1,33)$ & $0,29(0,67-1,26)$ & $0,64(0,12-3,41)$ & $0,64(0,12-3,41)$ \\
\hline \multicolumn{6}{|l|}{ Peso aos 12 meses } \\
\hline Adequado & & & & 0,07 & 0,07 \\
\hline Abaixo do peso & & & & $4,48(0,88-22,83)$ & $4,48(0,88-22,83)$ \\
\hline Acima do peso & & & & $0,65(0,14-2,97)$ & $0,65(0,14-2,97)$ \\
\hline Gravidade da cárie & & & & & $1,17(1,05-2,97) \quad 0,04$ \\
\hline
\end{tabular}

Modelo 1: Classe econômica $\mathrm{ABEP}^{39}$, cor da pele, escolaridade materna Modelo 2: Ajustado para as variáveis mantidas no modelo 1 (classe econômica $\mathrm{ABEP}^{39}$ ) + variáveis do modelo 2: cadastro em USF e inclusão no PBF. Modelo 3: Ajustado para as variáveis mantidas no modelo $1+$ variáveis do modelo 3: baixo peso ao nascer (BPN) e amamentação exclusiva até o $6^{\circ}$ mês de vida. Modelo 4: Ajustado para as variáveis mantidas no modelo 1 + variáveis do modelo 4: peso aos 12 meses. Modelo 5: Ajustado para as variáveis mantidas no modelo 1 + variáveis do modelo 5: gravidade da doença cárie e níveis séricos de albumina.

$\mathrm{RP}$, razão de prevalência. IC, intervalo de confiança.

Apenas as variáveis com valor significante $(\mathrm{p}<0,05)$ e mantidas $(\mathrm{p}<0,20)$ são descritas na tabela.

desnutrição ou dieta inadequada ${ }^{54}$. Entretanto, ajustando-se o modelo mais distalmente às variáveis socioeconômicas, não se conseguiu mostrar o efeito da classe econômica no desfecho.

Quanto à albumina, não houve significância na associação com o desfecho, discordando dos achados de Monte ${ }^{55}$, em que crianças com déficit de altura, apresentaram baixos níveis de albumina, comparadas com os níveis de albumina em crianças saudáveis.

Deve-se considerar que o presente estudo possui limitações. A baixa prevalência do desfecho teve implicações na precisão de algumas estimativas do estudo, com aumento da possibilidade de ocorrência de erro tipo II nos testes de hipótese. Apesar disso, foi possível identificar associação entre cárie dentária e deficiências na altura para a idade. Ademais, a restrição da amostra do estudo a crianças de classes sociais menos favorecidas, ao tempo em que reduziu a possibilidade de vieses de confundimento, pode ter implicações na validade externa do estudo. Assim, deve-se ter cautela ao extrapolar os resultados deste estudo para populações diferentes, com outras classes sociais ou faixas etárias.

Por se tratar de dados transversais da doença cárie, não podemos assumir que a gravidade da doença seja fator de risco para a deficiência nutricional, ou se essas alterações apenas coexistem por terem um fator causal comum, por exemplo, a qualidade da dieta das crianças. Ainda ou- tras investigações têm sugerido que deficiências nutricionais também poderiam ser fatores de risco para o aumento da ocorrência de cárie dentária $^{56-58}$ através de mecanismos como hipoplasia do esmalte, hipofunção da glândula salivar e mudanças na composição da saliva em quadros de desnutrição $0^{59-62}$. Dessa forma, o desenho do estudo não permite tratar adequadamente a questão da causalidade reversa.

No entanto, independente da temporalidade, o estudo evidencia associação entre a gravidade de lesões cariosas e o aumento do déficit antropométrico da altura para a idade, sugerindo a necessidade de políticas públicas intersetoriais.

Como atributo positivo deste estudo, destaca-se a utilização das curvas da $\mathrm{OMS}^{35}$ para classificação do estado antropométrico, uma vez que representa uma atualização das curvas do NCHS e incluem dados da população brasileira. Outro ponto que merece destaque foi a análise estatística baseada em modelo teórico hierarquizado, que permitiu avaliar e ajustar a associação entre cárie e desnutrição proteico-calórica para fatores mais distais, em coerência com o arcabouço teórico existente ${ }^{12,22,24,54}$.

A gravidade da doença cárie em crianças foi associada ao déficit antropométrico num modelo de ajustamento com confundidores mais clássicos dessa associação, sinalizando que a saúde bucal deve ser parte importante nas políticas públicas de atenção à infância. 


\section{Colaboradores}

MCB Silva, CMP Machado, MRC Ribeiro, EBAF Thomaz e CCC Ribeiro participaram igualmente de todas as etapas de elaboração do artigo.

\section{Agradecimentos}

À FAPEMA e à CNPq pelo suporte financeiro a pesquisa. Às crianças e seus responsáveis pela cooperação com a pesquisa.

\section{Referências}

1. Centro Latino-Americano e do Caribe de Informação em Ciências da Saúde. Descritores em Ciências da Saúde. São Paulo: Bireme; 2013. [acessado 2012 mar 20]. Disponível em: http://decs.bvs.br/cgi-bin/ wxis1660.exe/decsserver/.

2. Brasil. Ministério da Saúde (MS). Centro Brasileiro de Análise e Planejamento. Pesquisa Nacional de Demografia e Saúde da Mulher e da Criança PNDS 2006: RelatórioFinal. Brasília: MS; [página na Internet]. 2008. [acessado 2012 mar 20]. Disponível em: http://bvsms.saude.gov.br/bvs/pnds/img/relatorio_ final_pnds2006.pdf

3. Victora CG, Adair L, Fall C, Hallal PC, Martorell R, Richter L, Sachdev HS; Maternal and Child Undernutrition Study Group. Maternal and child undernutrition: consequences for adult health and human capital. Lancet 2008; 371(9609):340-357.

4. Onis M, Borghi MB, Borghi E. Prevalence and trends of stunting among pre-school children, 19902020. Public Health Nutr 2011; 15(1):142-148.

5. Monteiro CA, Benicio MHDA, Konno SC, Silva ACF, Lima ALL, Conde WL. Causes for the decline in child undernutrition in Brazil, 1996-2007. Rev Saude Publica 2009; 43(1):35-43.

6. Ferreira HS, Cesar JA, Assunção ML, Horta, BL. Time trends (1992-2005) in undernutrition and obesity among children under five years of age in Alagoas State, Brazil. Cad Saude Publica 2013; 29(4):793-800.

7. Onis M, Garza C, Victora CG, Onyango AW, Frongillo EA, Martines J. The WHO Multicentre Growth Reference Study: planning, study design, and methodology. Food Nutr Bull 2004; 25(Supl. 1):15-26.

8. Martins IS, Marinho SP, Oliveira DC, Araujo EAC. Pobreza, desnutrição e obesidade: inter-relação de estados nutricionais de indivíduos de uma mesma família. Cien Saude Colet 2007; 12(6):15531565.

9. American Academy of Pediatric Dentistry. Clinical guideline on baby bottle tooth decay/early childhood carie/ breastfeeding/ early childhood caries: unique challenges and treatment options. Academic Journal 2001; 23(7):29. (Pediatric Dentistry; Reference Manual 2001-2002)

10. Hallet KB. Early childhood caries and infant feeding practice. Community Dent Oral Epidemiol 2006; 19(4):237-242.

11. O'Rourke PK, Hallet KB. Pattern and severity of early childhood caries. Community Dent Oral Epidemiol 2006; 34(1):25-35.

12. Livny A, Fraiz FC. Oral health promotion promotion for schoolchildren- evaluation of a pragmatic approach with emphasis on improving brushing skills. BMC Oral Health 2007; 8:4.

13. Reisine S, Douglass JM. Psychosocial and behavioral issues in early childhood caries. Community Dent Oral Epidemiol 1998; 26:32-44.

14. Prakash P, Subramaniam P, Durgesh BH, Konde S. Prevalence of early childhood caries and associated risk factors in preschool children of Urban Bangalore, India: A cross-sectional study. Euro J Dent 2012; 6(2):141-152. 
15. Stecksén-Blicks C, Sunnegardh K, Borssén E. Caries experience and background factors in 4-year-old children: time trends 1967-2002. Caries Res 2004; 38(2):156-160

16. Mohebbi SZ, Virtanen JI, Vahid-Golpayegani M, Vehkalahti MM. Feeding habits as determinants of early childhood caries in a population where prolonged breastfeeding is the norm. Community Dent Oral Epidemiol 2008; 36(4):363-369.

17. Mobley C, Marshall TA, Milgrom P, Coldwell SE. The contribution of dietary factors to dental caries and disparities in caries. Acad Pediatr 2009; 9(6):410414.

18. Silva RP, Assaf AV, Mialhe FL. Diagnóstico de cárie dentária: uma visão além da boca para planejamento de ações em saúde coletiva. In: Pereira AC, organizador. Tratado de saúde coletiva em Odontologia. Nova Odessa: Napoleão; 2009. p. 315-340.

19. Sheihan A. Dental caries affects body weight, growth and quality of life in pre-school children. $\mathrm{Br}$ Dent J 2006; 201(10):625-626.

20. Acs G, Lodolini G, Kaminsky S, Cisneros GJ. Effect of nursing caries on body weight in a pediatric population. Pediatr Dent 1992; 14(5):302-305.

21. Moura MS, Moura LFAD, Mendes RF. Cárie dentária em crianças menores de cinco anos na cidade de Teresina - PI. Rev Odontol UNESP 2010; 39(3):143149.

22. Peres MA. Latorre MRDO, Sheiham A, Peres KGA, Barros FC, Hernandez PG, Maas AMN, Romano AR, Victora CG. Social and biological early life influenceson severity of dental caries in children aged 6 years. Community Dent Oral Epidemiol 2005; 33(1):53-63.

23. Norberg C, Hallström Stalin U, Matsson L, Thorngren-Jerneck K, Klingberg G. Body mass index (BMI) and dental caries in 5-year-old children from southern Sweden. Community Dent Oral Epidemiol 2012; 40(4):315-322.

24. Oliveira LB, Sheiham A, Bonecker M. Exploring the association of dental caries with social factors and nutritional status in Brazilian preschool children. Eur J Oral Sci 2008; 116(1):37-43.

25. Ngoenwiwatkul Y, Leela-Adisorn N. Effects of dental caries on nutritional status among first-grade primary school children. Asia Pac J Public Health 2009; 21(2):177-183.

26. Köksal E, Tekçiçek M, Yalçýn SS, Tuðrul B, Yalçýn S, Pekcan G Association between anthropometric measurements and dental caries in Turkish school children. Cent Eur J Public Health 2011; 19(3):147-151.

27. Prashanth ST, Venkatesh Babu, Vivek Dhruv Kumar, Amitha H. Comparison of Association of Dental Caries in Relation with Body Mass Index (BMI) in Government and Private School Children. J Dent Sci Res 2011; 2(2):1-5.

28. Ruhaya H, Jaafar N, Jamaluddin M, Ismail AR, Ismail NM, Badariah TC, Mat A, Mohamed SZ. Nutritional status and early childhood caries among preschool children in Pasir Mas, Kelantan, Malaysia. Arch Orofac Sci 2012; 7(2):1-7.

29. Chen W, Chen P, Chen SC, Shih WT, Hu HC. Lack of association between obesity and dental caries in three-year-old children. Acta Paed Sin 1998; 39(2): 109-111.
30. Macek MD, Mitola DJ. Exploring the association between overweight and dental caries among US children. Pediatr Dent 2006; 28(4):375-380.

31. Kopycka-Kedzierawski DT, Auinger P, Billings RJ, Weitzman M. Caries status and overweight in 2- to 18-year-old US children: findings from national surveys. Community Dent Oral Epidemiol 2008; (36):157-167.

32. Sheller B, Churchill SS, Williams BJ, Davidson B. Body Mass Index of Children With Severe Early Childhood Caries. Pediatri Dent 2009; 31(3):217-221.

33. Carmo CDS, Alves CMC, Cavalcante PR, Ribeiro CCC. Evaluation of fluoride levels in the public water supply in São Luis Island, Maranhão State, Brazil. Cien Saude Colet 2010; 15(Supl. 1):1835-1840.

34. Silva MCB, Silva RA, Ribeiro CCC, Cruz MCN: Profile of public dental care for children and adolescents in São Luis, Maranhão State. Cien Saude Colet 2007; 12(5):1237-1246.

35. World Health Organization (WHO). Child growth standards: length/height-for-age, weight-forage, weight-for-length, weight-for-height and body mass indexfor-age. Methods and development. Geneva: WHO; 2006.

36. World Health Organization (WHO). WHO Anthro [computer program]. Version 3.2.2. Genebra: WHO; 2006.

37. World Health Organization (WHO). WHO Anthro Plus [computer program]. Version 1.4.0. Genebra: WHO; 2006.

38. World Health Organization (WHO). Oral health surveys. Basic methods, $4^{\text {th }}$ Edition. Geneva: WHO; 1997.

39. Associação Brasileira de Estudos Populacionais (ABEP). Critério de Classificação Econômica Brasil. 2012, 1:3.[página na Internet]. [acessado $2012 \mathrm{fev}$ 23]. Disponível em http://www.abep.org.br

40. World Health Organization (WHO). Meeting of advisory group on Maternal nutrition and Low birthweight Geneva: WHO; [página na Internet]2002. [acessado 2012 out 17]. Disponível em: http://www. who.int/nutrition/.../advisory_group_lbw.pdf

41. STATA [computer program]. Version 10.0. Texas: Stata Corp. College Station; 2007.

42. Blackburn GL, Harvey KB. Nutritional assessment as a routine in clinical medicine. Postgrad Med 1982; 71(5):46-63.

43. Costa AAA, organizadora. Creche comunitária, uma alternativa popular. Salvador: NEIM, UFMA; 1991.

44. Santos DJS, Palomares NB, Normando D, Quintão CCA. Raça versus etnia: diferenciar para melhor aplicar. Dental Press J Orthod, 2010; 15(3):121-124.

45. Santos JAF. Classe Social e Desigualdade de Saúde no Brasil. RBCS 2011; 26(75):27-51.

46. Lima ALL, Silva ACF, Konno SC, Conde WL, Benício MLD, Monteiro CA. Causas do declínio acelerado da desnutrição infantil no Nordeste do Brasil (19861996-2006). Rev Saude Publica 2010; 44(1):17-27.

47. Coimbra LC, Pereira OS, Lopes MLH, Fonseca LMB, Alencar MN, Wanderlei, MIG. Indicadores operacionais da atenção básica em São Luís, Maranhão. Rev Pesq Saúde 2012; 13(2):38-41.

48. Victora CG, de Onis M, Hallal PC, Blössner M, Shrimpton R. Worldwide timing of growth faltering: revisiting implications for interventions. Pediatric 2010; 125(3):473-480. 
49. Ayhan H, Suskan E, Yildirim S. The effect of nursing or rampant caries on height, body weight and head circumference. J Clin Pediatr Dent 1996; 20(3):209-212.

50. Floyd B. Associations between height, body mass, and frequency of decayed, extracted, and filled deciduous teeth among two cohorts of Taiwanese first graders. Am J Phys Anthropol 2009; 140(1):113-119.

51. Germain L. Differential diagnosis of toothache pain. Part I, Odontogenic etiologies. Dent Today 2012; 31(7):92-97.

52. Sheiham A. Dental caries affects body weight, growth and quality of life in pre-schoolchildren. $\mathrm{Br}$ Dent $J$ 2006; 201(10):625-626.

53. Cypriano S, Hugo FN, Sciamarelli MC, Tôrres LHN, Sousa MLR, Wada RS. Fatores associados à experiência de cárie em escolares de um município com baixa prevalência de cárie dentária. Cien Saude Colet 2011; 16(10):4095-4106.

54. Fonseca MA. The effects of poverty on children's development and oral health. Pediatr Dent 2012; 34(1):32-38.

55. Monte CMG. Desnutrição: um desafio secular à nutrição infantil. J Pediatr 2000; 76(Supl. 3):s285s297.

56. Lueangpiansamut J, Chatrchaiwiwatana S, Muktabhant B, Inthalohit W. Relationship between dental caries status, nutritional status, snack foods, and sugar-sweetened beverages consumption among primary schoolchildren grade 4-6 in Nongbua Khamsaen school, NaKlang district, Nongbua Lampoo Province, Thailand. J Med Assoc Thai 2012; 95(8):1090-1097.

57. Sheetal A, Hiremath VK, Patil AG, Sajjansetty S, Kumar SR. Malnutrition and its oral outcome - a review. J Clin Diagn Res 2013; 7(1):178-180.

58. Delgado-Angulo EK, Hobdell MH, Bernabé E. Childhood stunting and caries increment in permanent teeth: a three and a half year longitudinal study in Peru. Int J Paediatr Dent 2013; 23(2):101-109.

59. Alvarez J. Nutrition, tooth development, and dental caries. Am J Clin Nutr 1995; 61(Supl.):410S-416S.

60. Psoter WJ, Reid BC, Katz RV. Malnutrition and Dental Caries: A Review of the Literature. Caries Res 2005; 39(6):441-447.

61. Russell SL, Psoter WJ, Charles GJ, Prophte S, Gebrian B. Protein-energy malnutrition during early childhood and periodontal disease in the permanent dentition of Haitian adolescents aged 12-19 years: a retrospective cohort study. Int J Paediatr Dent 2010; 20(3):222-229.

62. Psoter WJ, Spielman AL, Gebrian B, Rudolph SJ, Katz RV. Effect of childhood malnutrition on salivary flow and pH. Arch Oral Biol 2008; 53(3):231237.

Artigo apresentado em 28/05/2013

Aprovado em 30/06/2013

Versão final apresentada em 26/07/2013 